\title{
Is cyclooxygenase-2 the alpha and the omega in cancer?
}

\section{Stephen M. Prescott}

Huntsman Cancer Institute, University of Utah, 2000 Circle of Hope, Salt Lake City, Utah 84112-5550, USA.

Phone: (801) 585-3401; Fax: (801) 585-6345; E-mail: steve.prescott@hci.utah.edu.

Angiogenesis, the development of new blood vessels, is an essential step in the growth of tumors, since the growth of the malignant cells is limited by the availability of nutrients. Recent studies, including one by Williams et al. in this issue of the JCI (1), show that angiogenesis requires cyclooxygenase-2 (COX-2), the enzyme that catalyzes the committed step in the synthesis of prostaglandins. This exciting development suggests that inhibitors of COX-2 may be useful in the treatment of even malignant cancers and may not be restricted to use as chemopreventive agents; the latter application is currently being tested. As with most interesting findings, this report generates at least as many questions as it answers. In recent studies, a number of groups agree that COX-2 is crucial for tumor angiogenesis, but their agreement ends here - is the essential COX-2 found in the carcinomatous epithelial cells, the stromal cells, or the endothelial cells of the neovasculature? The answer, as indicated in Figure 1, seems to be "all of the above". In earlier work, some of the authors of the current report (1) used an in vitro system to examine the role(s) of COX-2 in angiogenesis and reported that colon cancer cells expressing COX-2 induced an angiogenic response in cocultured endothelial cells (2). In this earlier work, they also examined tumor cell xenografts and found that expression of COX-2 by the tumor cells was a key determinant of tumor growth. The implanted cell line grew poorly if there was no COX-2 expression, while cells expressing COX-2 grew to much larger tumors, whose growth could be blocked with a COX-2 inhibitor. In the same study, they found that COX-1 from the host was also important, and they implicated endothelial cells as the target. More recently, Sawaoka et al. implanted $\mathrm{COX}-2^{+}$or COX-2- tumor cells into mice, which were then treated with inhibitors of COX-1 or -2 (3). When the implanted carcinoma cells were COX-2 $2^{+}$, selective inhibitors of COX-2 decreased angio- genesis and tumor growth. However, when the implanted cells were COX-2-, the selective COX-2 inhibitors had no effect on tumor volume or on angiogenesis index. This result confirmed the findings of Tsujii et al. (2) that COX-2 expression in the cancer cell is key to angiogenesis. Likewise, Sawaoka and colleagues found that COX-1 from the host, presumably in endothelial cells, was also required for a complete response (3).

The COX field has a record of turning up complications and controversy just when things seem to be clear, and COX function in angiogenesis is no exception. Jones et al. (4) recently reported that a selective inhibitor of COX-2 could block the angiogenic response to growth factors by cultured endothelial cell. This report conflicted with the earlier work suggesting that COX-1 was responsible for the endothelial cell response, and, further, it suggested a different locus for the responsible COX-2 in tumors. In support of this view, Masferrer and coworkers reported that multiple human cancers express COX-2 in the endothelial cells of the neovasculature, as well as the epithelial cells and some inflammatory cells (5). These authors also found that a selective inhibitor of COX-2, but not a selective inhibitor of

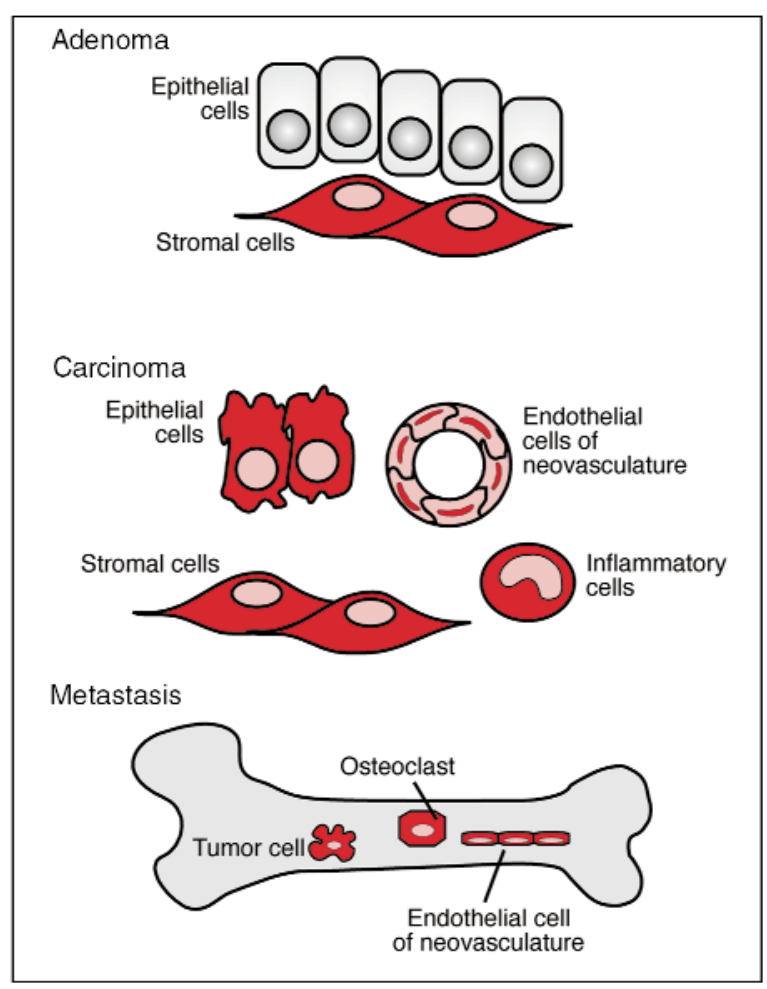

\section{Figure 1}

COX-2 expression in multiple cells in tumors of different stages. The sequence of events shown has been constructed from studies with multiple types of cancer. For example, the work on the earliest events was from mouse and human experiments on adenomatous polyps of the colon, in which the earliest expression of COX-2 (shown in red) is in the stromal cells. In several types of cancers, COX-2 is found in multiple cells in the late stages. Metastatic tumors in bone and various other organs express COX- 2 in the metastatic cells and surrounding cells. In the case of bone, osteoclasts, which degrade the bone matrix and allow growth of the newly metastasized tumor, are among these COX $-2^{+}$cells. 
Stromal cell

(upstream)

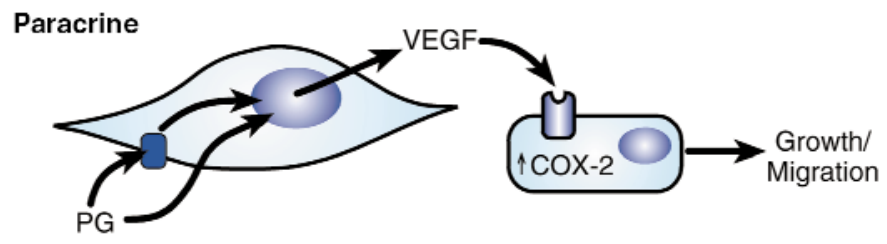

Autocrine
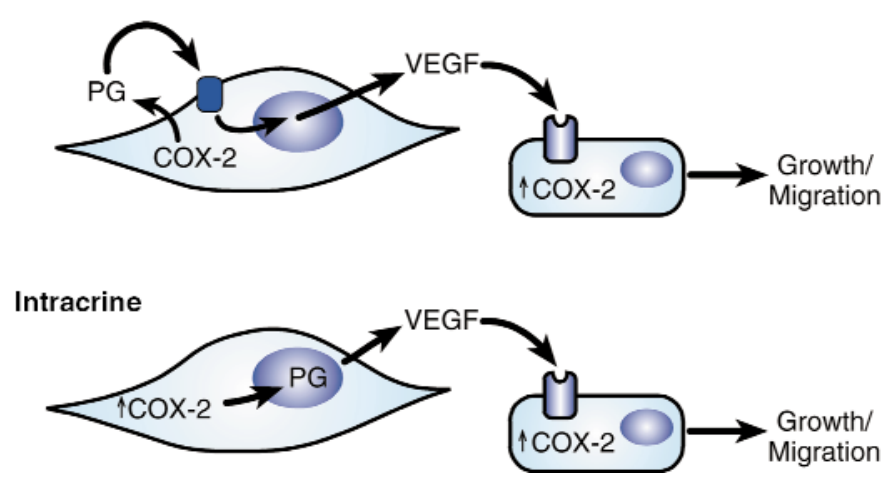

Figure 2

Possible routes of prostaglandin signaling in the induction of tumor angiogenesis. The experiments by Williams (1) suggest that COX-2 (and presumably prostaglandin synthesis) is required in the upstream stromal cells that provide VEGF to endothelial cells. Other investigators have found that COX-2 is required in the endothelial cells that will form the new blood vessels (downstream). Prostaglandins can exert their effects either through receptors on the cell surface, which are coupled to $G$ proteins and various intracellular signaling pathways, or by the nuclear transcription factors (peroxisome proliferator-activated receptors). The routes through which prostaglandins act in this pathway are not known, but several possibilities are shown. In paracrine signaling, exogenous but locally produced prostaglandins (PG) act on the stromal cell to induce VEGF expression. Autocrine signaling differs in that the prostaglandins are produced in stromal cells and are secreted and act on receptors (blue) on the surface of these same cells. In intracrine signaling, prostaglandins also act on the producing cell, but they do so prior to being secreted. Similar events not shown here could occur in the downstream endothelial cell as well.

COX-1, blocked angiogenesis and tumor growth in an implantation model. Based on these experiments, they proposed that COX-2 in host endothelial cells is the target of antiangiogenic therapy, and they employed a corneal model to show that selective inhibition of COX-2 blocks growth factor-dependent angiogenesis. Using a similar corneal model, as well as in vitro experiments, Daniel et al. (6) also concluded that endothelial cell COX-2 acts at an essential step in angiogenesis, and experiments in a different corneal model yielded the same conclusion (7). The expression of COX-2 by endothelial cells was no surprise, as some of the earliest work on human COX-2 expression used these cells $(8,9)$, but the idea that it contributes to angiogenesis was novel.

Prior to the present study (1), the outstanding unanswered questions about COX function in this pathway centered on cell- and enzyme subtype-specifici- ty: Does COX-2 act in the cancer cell, the endothelial cell, or both to control angiogenesis? Does COX-1 contribute as well? The experimental approach of Williams et al. (1) appears ideally suited to address these questions. They implanted Lewis lung carcinoma cells, which generate COX $-2^{+}$tumors, into mice that had been genetically engineered to lack either COX-2 or COX-1, and they observed a dramatic suppression of tumor growth and angiogenesis in the animals that lacked COX-2, compared to wild-type mice. The presence ence for tumor growth. Williams et al. conclude that COX-2 is required - as everyone now appears to agree - but that it is the COX-2 in the host cells, not the tumor, that matters for tumor growth. Furthermore, they conclude that the relevant COX-2 is expressed not in endothelial cells but in the stroor absence of COX-1 made no differ- mal cells of the tumor. This latter claim is based, first, on the observation that COX-2 occurs in stromal cells, and second, on these researchers' work with fibroblasts cultured from animals lacking COX expression. Because COX2-deficient fibroblasts failed to secrete VEGF, an important component of the angiogenic response, the current work identifies a previously unsuspected step in tumor angiogenesis, whereby COX-2 activity in the upstream stromal cell is required to deliver VEGF to the downstream endothelial cell for the latter to generate a new blood vessel.

\section{Precedents and problems}

Abundant evidence indicates that inhibition of COX protects against cancer, particularly colon cancer (10). For example, in animal studies COX inhibitors slowed or prevented colon cancer in different models, and in epidemiological studies chronic intake of COX inhibitors (nonsteroidal anti-inflammatory drugs) lowered the risk of colon cancer and polyps. Furthermore, a variety of studies showed that COX-2, in particular, is overexpressed in colon and other cancers (10). COX-1, in contrast, is expressed constitutively at a low level in the normal tissues and does not increase during transformation. In another study, mice with a mutated $A p c$ gene were protected from the development of adenomatous polyps if they also had COX-2 knocked out or if they were treated with a selective inhibitor of COX-2 (11). Since these polyps are precursors to cancer, this study indicated that COX-2 acts at a rate-limiting step and contributes at an early stage of the disease. Many subsequent studies with inhibitors of COX-2, in mice and humans, have supported this conclusion. Nevertheless, the report of Oshima et al. (11) contained a surprise: COX-2 was expressed not in the colon epithelial cells but rather in the stromal cells. This was surprising because Apc has been thought to act by cell-autonomous mechanisms such that loss of adenomatous polypsis coli function within the colon epithelial cell leads to deranged patterns of growth and/or apoptosis. The work of Oshima and colleagues suggested that the sequence might not be so straightforward, and that stromal cells are essential even at the earliest stages of a polyp. How increased COX-2 expression might be induced in the stroma in early carcinogenesis remains unanswered. 
One puzzling feature of the present results from Williams et al. (1) is that the COX-2 in the implanted tumor cells did not support angiogenesis. The prostaglandins derived from the actions of COX typically are secreted and then bind to receptors either on the same cell (autocrine signaling) or on adjacent cells (paracrine signaling) (Figure 2), providing what Kinzler and Vogelstein described as a "landscaping" effect (also known as a field effect) (12). Given the multiple cells in which COX2 appears during carcinogenesis, it may be that prostaglandins exert a positive (feed-forward) effect, inducing expression of COX-2 in nearby cells. We have shown that some prostaglandins, added exogenously, induced COX-2 expression in cultured epithelial cells (13), suggesting a possible mechanism for such a sequence of events. But if prostaglandins signal between cells during carcinogenesis, it remains unclear why tumor cell-derived COX-2 fails to support VEGF secretion by the stromal cells in the experiments by Williams et al. (1). Other studies have shown that prostaglandins can induce VEGF secretion in target cells $(14,15)$, and the stromal cells in tumors have active transcription of the VEGF gene (16). In contrast, Williams et al. seem to find that synthesis and secretion of VEGF by stromal cells require that the prostaglandin be produced within the same cell - an example of what has been called intracrine signaling (Figure 2 ) - since fibroblasts from the COX2-deficient mice did not secrete VEGF. There is a caveat, however, in that the investigators have not yet determined whether stimulating fibroblasts with either exogenous prostaglandins or other agonists, such as growth factors, can increase the secretion of VEGF (R.N. DuBois, personal communication). It would be surprising if COX-2 is indeed required within the cell that synthesizes the endothelial cell growth factors, but such a finding might be explained by the action of endogenous prostaglandins on intracellular receptors, such as the peroxisome proliferator-activated receptors. On the other hand, if COX-2 is not required within the cell, the failure of endogenous tumor cell-derived COX-2 to support angiogenesis might be explained by insufficient production of prostaglandins by the upstream stromal cells. Induction of COX-2 by cytokines, growth factors, or tumor promoters usually results in a substantial increase in the capacity of tissues to synthesize and release prostaglandins, in part because other players in the pathway, such as phospholipase, may be activated as well. Perhaps this induction occurs less efficiently in this model. This possibility is attractive in that it may explain why COX-2 seems to be more important than COX-1; detailed biochemical studies have failed to reveal substantial differences between them except for the fact that COX-2 can be induced to high levels.

In summary, evidence from a variety of sources suggests that COX-2 acts early in colon carcinogenesis and that inhibitors of COX-2, or deficiency in COX-2, block the formation of adenomatous polyps or cause them to regress. At early stages of carcinogenesis, COX-2 in the intestine appears to be expressed largely in stromal cells, but it is later found in inflammatory cells in or near the tumor, in the endothelial cells of the neovasculature, and in the carcinomatous epithelial cells. The weight of evidence from these recent studies shows that COX-2 in endothelial cells is required for angiogenesis, but the present work of Williams et al. (1) strongly suggests that COX-2 is also required upstream to support the secretion of growth factors for the endothelial cells. Likewise, expression in the carcinomatous epithelial cells appears to confer growth advantages to the tumor, perhaps because prostaglandins activate the secretion of growth factors from stromal cells, which then stimulate the endothelial cells. Finally, expression of COX-2 may contribute to the growth of metastatic tumors (Figure 1): Prostaglandin $E_{2}$ is well known to stimulate the remodeling of bone by osteoclasts, allowing metastatic tumors to thrive, and recent studies have shown that COX-2 is induced in the osteoclasts and the neovasculature in the bone. Thus, investigation today suggests that COX-2 may play important roles from the earliest to the latest stages of tumorigenesis and its consequences. Whether prostaglandins act in a paracrine fashion to exert a "field" or "landscaping" effect or in a cell-autonomous fashion remains to be resolved, but if the pattern of work in this field continues, perhaps the answer will be "both."

1. Williams, C.S., Tsujii, M., Reese, J., Dey, S.K., and DuBois, R.N. 2000. Host cyclooxygenase- 2 modulates carcinoma growth. J. Clin. Invest. 105:1589-1594

2. Tsujii, M., et al. 1998. Cyclooxygenase regulates angiogenesis induced by colon cancer cells. Cell. 93:705-716.

3. Sawaoka, H., et al. 1999. Cyclooxygenase inhibitors suppress angiogenesis and reduce tumor growth in vivo. Lab. Invest. 79:1469-1477.

4. Jones, M.K., et al. 1999. Inhibition of angiogenesis by nonsteroidal anti-inflammatory drugs: insight into mechanisms and implications for cancer growth and ulcer healing. Nat. Med. 5:1418-1423.

5. Masferrer, J.L., et al. 2000. Antiangiogenic and antitumor activities of cyclooxygenase-2 inhibitors. Cancer Res. 60:1306-1311.

6. Daniel, T.O., Liu, H., Morrow, J.D., Crews, B.C., and Marnett, L.J. 1999. Thromboxane A2 is a mediator of cyclooxygenase-2-dependent endothelial migration and angiogenesis. Cancer Res. 59:4574-4577.

7. Yamada, M., Kawai, M., Kawai, Y., and Mashima, Y. 1999. The effect of selective cyclooxygenase-2 inhibitor on corneal angiogenesis in the rat. Curr. Eye Res. 19:300-304.

8. Jones, D.A., Carlton, D.P., McIntyre, T.M., Zimmerman, G.A., and Prescott, S.M. 1993. Molecular cloning of human prostaglandin endoperoxide synthase type II and demonstration of expression in response to cytokines. J. Biol. Chem. 268:9049-9054

9. Hla, T., and Neilson, K. 1992. Human cyclooxygenase-2 cDNA. Proc. Natl. Acad. Sci. USA. 89:7384-7388.

10. Prescott, S.M., and Fitzpatrick, F.A. 2000. Cyclooxygenase-2 and carcinogenesis. Biochim. Biophys. Acta. 1470:M69-M78.

11. Oshima, M., et al. 1996. Suppression of intestinal polyposis in $A p c^{\Delta 716}$ knockout mice by inhibition of prostaglandin endoperoxide synthase-2 (COX-2). Cell. 87:803-809.

12. Kinzler, K.W., and Vogelstein, B. 1998. Landscaping the cancer terrain. Science. 280:1036-1037.

13. Meade, E.A., McIntyre, T.M., Zimmerman, G.A. and Prescott, S.M. 1999. Peroxisome proliferators enhance cyclooxygenase- 2 expression in epithelial cells. J. Biol. Chem. 274:8328-8334.

14. Hoper, M.M., et al. 1997. Prostaglandins induce vascular endothelial growth factor in a human monocytic cell line and rat lungs via cAMP. Am.J. Respir. Cell. Mol. Biol. 17:748-756.

15. Cheng, T., Cao, W., Wen, R., Steinberg, R.H., and LaVail, M.M. 1998. Prostaglandin E2 induces vascular endothelial growth factor and basic fibroblast growth factor mRNA expression in cultured rat Muller cells. Invest. Ophthalmol. Vis. Sci. 39:581-591.

16. Fukumura, D., et al. 1998. Tumor induction of VEGF promoter activity in stromal cells. Cell. 94:715-725. 